Revue d'histoire de l'Amérique française

Q4. REVUE D.HISTOIRE DE L'AMÉRIQUE FRANÇAISE

\title{
Les institutrices rurales du Bas-Canada : incompétentes et inexpérimentées?
}

\section{Andrée Dufour}

Volume 51, numéro 4, printemps 1998

URI : https://id.erudit.org/iderudit/005404ar

DOI : https://doi.org/10.7202/005404ar

Aller au sommaire du numéro

Éditeur(s)

Institut d'histoire de l'Amérique française

ISSN

0035-2357 (imprimé)

1492-1383 (numérique)

Découvrir la revue

Citer cet article

Dufour, A. (1998). Les institutrices rurales du Bas-Canada : incompétentes et inexpérimentées? Revue d'histoire de l'Amérique française, 51(4), 521-548. https://doi.org/10.7202/005404ar
Résumé de l'article

Cet article entend projeter une image plus précise des institutrices ayant travaillé dans les écoles de campagne du Bas-Canada de 1825 à 1860. On y soutient que le processus de féminisation du métier d'enseignant a été trop rapidement attribué aux maigres salaires consentis à de toutes jeunes filles, jugées à tort inexpérimentées, incompétentes et passives. Appuyée sur des sources nouvelles, l'analyse révèle un processus plus complexe qui s'amorce très tôt au Québec, dès le premier tiers du XIXe siècle, et qui tient davantage des difficultés financières et des préférences des communautés locales, de l'appropriation du métier par les institutrices, du retrait des hommes de l'enseignement primaire et des politiques des autorités scolaires. Possédant souvent une expérience de plusieurs années ainsi qu'une formation jugée adéquate et manifestant un réel souci d'améliorer leurs connaissances et leur pratique, les institutrices rurales ont apporté une contribution essentielle à l'entreprise de scolarisation de l'ensemble de la jeunesse bascanadienne.
Tous droits réservés @ Institut d'histoire de l'Amérique française, 1998
Ce document est protégé par la loi sur le droit d'auteur. L'utilisation des services d'Érudit (y compris la reproduction) est assujettie à sa politique d'utilisation que vous pouvez consulter en ligne.

https://apropos.erudit.org/fr/usagers/politique-dutilisation/ 


\title{
LES INSTITUTRICES RURALES DU BAS-CANADA: INCOMPÉTENTES ET INEXPÉRIMENTÉES?
}

\author{
ANDRÉE DUFOUR \\ Département de sciences humaines \\ Cégep Saint-Jean-sur-Richelieu
}

\begin{abstract}
RÉSUMÉ
Cet article entend projeter une image plus précise des institutrices ayant travaillé dans les écoles de campagne du Bas-Canada de 1825 à 1860. On y soutient que le processus de féminisation du métier d'enseignant a été trop rapidement attribué aux maigres salaires consentis à de toutes jeunes filles, jugées à tort inexpérimentées, incompétentes et passives. Appuyée sur des sources nouvelles, l'analyse révèle un processus plus complexe qui s'amorce très tôt au Québec, dès le premier tiers du XIX ${ }^{\mathrm{e}}$ siècle, et qui tient davantage des difficultés financières et des préférences des communautés locales, de l'appropriation du métier par les institutrices, du retrait des hommes de l'enseignement primaire et des politiques des autorités scolaires. Possédant souvent une expérience de plusieurs années ainsi qu'une formation jugée adéquate et manifestant un réel souci d'améliorer leurs connaissances et leur pratique, les institutrices rurales ont apporté une contribution essentielle à l'entreprise de scolarisation de l'ensemble de la jeunesse bascanadienne.
\end{abstract}

\begin{abstract}
This article wishes to draw a more accurate portrait of the women who taught in the rural schools of Lower Canada between 1825 and 1860. It argues that the feminization of teaching has been ascribed too rapidly to the lower salaries paid to very young girls, who were wrongly perceived as inexperienced, incompetent and passive. Based on new sources, this study presents feminization as a more complex process, which began in Quebec as early as the first third of the $19^{\text {th }}$ century, and which is more contingent on factors such as the preferences and financial difficulties of the local communities, the appropriation of the occupation by women, the withdrawal of men from elementary school teaching and the politics of local school authorities. Having often acquired several years of experience as well as what was deemed to be adequate training, genuinely concerned with the improvement of their knowledge and skills, women rural school teachers have made an essential contribution to the schooling of youth in Lower Canada.
\end{abstract}

RHAF, vol. 51, n 4, printemps 1998 
Plus de trente ans se sont écoulés depuis la parution des travaux d'André Labarrère-Paulé sur les instituteurs et les institutrices laïques du Québec au XIX ${ }^{\mathrm{e}}$ siècle $^{1}$. L'image qui s'en dégage est celle de maîtres le plus souvent incompétents et sous-payés. Seuls les instituteurs des villes échappent à ce noir portrait puisqu'ils apparaissent généralement instruits, dirigeant des écoles réputées et participant à la formation des maîtres. Le bilan est dans l'ensemble accablant, particulièrement pour les institutrices de campagne. Celles-ci sont décrites en des termes fort peu flatteurs: «peu instruites», «le plus souvent incompétentes», «inexpérimentées et inertes», peu soucieuses d'améliorer leurs connaissances et leur pratique. «Très jeunes» pour la plupart, elles acceptent des rémunérations bien inférieures à celles des hommes et contribuent ainsi à une féminisation «catastrophique» du métier. Concurrentes déloyales des instituteurs, seuls à faire preuve de combativité et d'une volonté de valoriser le métier, elles s'avèrent en somme «une entrave aux progrès de la classe enseignante ${ }^{2}$ ».

Plusieurs historiennes ont réagi à ce sombre tableau et ont projeté de l'enseignante une image plus nuancée ${ }^{3}$. On pourrait dès lors estimer que la question s'en trouve réglée. Deux raisons m'incitent pourtant à revenir sur le sujet. La perception négative des femmes dans l'enseignement demeure ${ }^{4}$. Et, surtout, ces études concernent expressément la fin du XIX ${ }^{\mathrm{e}}$ siècle et le $\mathrm{XX}^{\mathrm{e}}$ siècle ${ }^{5}$, un tout autre contexte marqué notamment par la

1. André Labarrère-Paulé, Les instituteurs lä̈ques au Canada français, 1836-1900 (Québec, Les Presses de l'Université Laval, 1965). L'auteur avait d'abord publié un premier article sur le sujet en 1963 dans The Canadian Historical Review, qui a été repris sous le titre «L'instituteur laïque canadien-français au $19^{\mathrm{e}}$ siècle», dans Marcel Lajeunesse, dir., L'éducation au Québec, $19^{e}$ et $20^{e}$ siècles (Montréal, Boréal Express, 1971), 59-76.

2. Voir dans André Labarrère-Paulé, Les instituteurs lä̈ques..., 133, 152, 200, 299 et 354 surtout.

3. Notamment Marîse Thivierge, Les institutrices rurales à l'école primaire catholique au Québec de 1900 à 1964, thèse de doctorat, Université Laval, 1981; Marta Danylewycz, «Sexes et classes sociales dans l'enseignement: le cas de Montréal à la fin du $19^{\mathrm{e}}$ siècle», dans Nadia FahmyEid et Micheline Dumont, Maîtresses de maison, maîtresses d'école. Femmes, famille et éducation dans l'histoire du Québec (Montréal, Éditions du Boréal Express, 1983), 93-118; Marta Danylewycz, Beth Light et Alison Prentice, «The Evolution of the Sexual Division of Labour Teaching: a Nineteenth-Century Ontario and Quebec Case Study», Histoire sociale/Social History, 16,3 (mai 1983): 81-109. Mentionnons également Ruby Heap, «Les femmes laïques au service de l'enseignement public catholique à Montréal: les écoles des "dames et demoiselles"», Canadian Woman Studies/Les cahiers de la femme, 7,3 (1986): 55-60.

4. On la retrouve chez Fernand Ouellet dans «La question sociale au Québec, 1880-1930: la condition féminine et le mouvement des femmes dans l'historiographie», Histoire sociale/Social History, 21,42 (novembre 1988): 328-332.

5. Seul Jean-Pierre Charland dans «L'éducation par l'exemple: le contrôle des comportements des instituteurs et des institutrices des écoles publiques québécoises, 1842-1897», dans Yves Roby et Nive Voisine, dir., Érudition, humanisme et savoir (Sainte-Foy, Les Presses de l'Université Laval, 1996), 195-214, aborde quelque peu la condition des institutrices rurales. 
forte présence de l'Église et l'existence d'un système scolaire bien établi. Il m'apparaît donc pertinent de tracer un portrait plus précis de la présence des institutrices laïques dans les écoles rurales de la première moitié du $\mathrm{XIX}^{\mathrm{e}}$ siècle, lorsque débute l'entreprise étatique de scolarisation de toute la jeune population bas-canadienne. J'examinerai à cette fin leur formation, leur expérience dans l'enseignement et leur attitude vis-à-vis de leur métier. Une historiographie plus récente et mes propres recherches, effectuées à partir des enquêtes sur l'éducation au XIX ${ }^{\mathrm{e}}$ siècle, des rapports des surintendants de l'instruction publique et des inspecteurs, ainsi que de la correspondance adressée aux autorités scolaires, entre autres par les mâ̂tres et les commissaires d'école, seront mises à profit ${ }^{6}$. Ces sources permettent, dans un premier temps, de revenir sur le rythme et l'origine de la féminisation du corps enseignant au Bas-Canada ${ }^{7}$.

\section{RYTHME ET ORIGINE DE LA FÉMINISATION DU CORPS ENSEIGNANT BAS-CANADIEN}

La féminisation du corps enseignant constitue un processus commun en Amérique du Nord qui s'est généralement produit à partir de la seconde moitié du XIX ${ }^{\mathrm{e}}$ siècle. Précisons d'entrée de jeu que, par féminisation, j'entends cette tendance selon laquelle les femmes occupent une place croissante dans une sphère d'activité donnée, dans l'enseignement en l'occurrence. Ainsi, en Amérique, les femmes deviennent majoritaires dans l'enseignement primaire durant le dernier quart du $\mathrm{XIX}^{\mathrm{e}}$ siècle $^{8}$.

Le cas du Québec diffère toutefois de ce modèle. L'arrivée massive des femmes dans l'enseignement s'y est produite beaucoup plus tôt, en fait dès la première moitié du XIX ${ }^{\mathrm{e}}$ siècle. André Labarrère-Paulé a situé l'événement de façon approximative. Il écrit: «Les instituteurs ne sont pas très inférieurs en nombre aux institutrices en $1836^{\circ} »$ et ajoute «La féminisation de l'enseignement, [est] nettement affirmée depuis $1846^{10} . \gg$ Par la suite, l'historienne Alison Prentice a souligné la spécificité du Québec dans le

6. On trouvera une critique de la plupart de ces sources dans ma thèse de doctorat, La scolarisation au Bas-Canada, 1826-1859: une interaction État-communautés locales, Université du Québec à Montréal, 1992, 45-49.

7. Comme on le sait, le Bas-Canada devient officiellement la section est de la Province du Canada (le Canada-Uni) en 1840. Je préfère cependant conserver pour la partie de l'étude qui concerne la période sous l'Union, l'appellation Bas-Canada, appellation qui survit d'ailleurs dans l'usage populaire et même dans les documents de source gouvernementale.

8. Voir notamment à ce propos Alison Prentice, «The Feminization of Teaching», dans Susan Mann Trofimenkoff et Alison Prentice, dir., The Neglected Majority: Essays in Canadian Women's History (Toronto, McClelland and Stewart, 1977), 51.

9. André Labarrère-Paulé, Les instituteurs lä̈ques..., 93.

10. André Labarrère-Paulé, «L'instituteur laïque»..., 65. 
rythme de la féminisation de l'enseignement primaire. En 1851, fait-elle remarquer, les femmes du Bas-Canada y sont déjà majoritaires, alors que dans le Haut-Canada et dans la province de la Nouvelle-Écosse, par exemple, la proportion d'hommes dans l'enseignement s'élève à plus de $75 \%{ }^{11}$. Dans une brève synthèse, sur l'instruction des filles au Québec, Micheline Dumont rappellait que «La féminisation du personnel enseignant, phénomène maintenant bien connu des sociétés nord-américaines, s'est produite au Québec beaucoup plus tôt que partout ailleurs ${ }^{12}$.» De fait, seul Fernand Ouellet se démarque en faisant état d'une «écrasante» supériorité masculine chez les enseignants à la fin du premier tiers du $\mathrm{XIX}^{\mathrm{e}}$ siècle $^{13}$.

Les premiers résultats de mes recherches sur les maîtres et les maitresses d'école du Bas-Canada font aussi apparaître une féminité ${ }^{14}$ déjà acquise $\mathrm{du}$ corps enseignant dès les premières décennies du $\mathrm{XIX}^{\mathrm{e}}$ siècle, en ce qui concerne l'île de Montréal ${ }^{15}$. En effet, l'analyse de deux recensements des écoles de l'île, effectués par Jacques Viger en 1825 et en $1835^{16}$, révèle une féminité de $58 \%$, en 1825 , et de $64 \%$, en 1835 , du personnel enseignant. L'île de Montréal était toutefois formée alors d'une nette majorité de citadins, résidant dans la vieille ville et les faubourgs de la cité de Montréal, et d'une population rurale relativement peu nombreuse ${ }^{17}$, dispersée dans tout le reste de l'île, soit dans les paroisses de Longue-Pointe, Pointe-aux-Trembles, Rivière-des-Prairies, Sault-au-Récollet, Saint-Laurent, Sainte-Gene-

11. Alison Prentice, loc. cit., 55.

12. Micheline Dumont, L'instruction des filles au Québec (1639-1960) (Ottawa, Société historique du Canada, coll. «Brochure historique», nº 49, 1990), 15.

13. Fernand Ouellet, loc. cit., 328-329. L'auteur arrive toutefois à ce constat par une analyse bien superficielle des sources, que j'ai remise en question dans «Un commentaire sur une prétendue supériorité masculine écrasante chez les enseignantes bas-canadiennes du premier tiers du $19^{\mathrm{e}}$ siècle», Histoire sociale/Social History, 23,45 (mai 1990): 114-116.

14. J'emploie l'expression féminité pour souligner la composition majoritairement féminine du métier.

15. Ces résultats ont été communiqués lors de deux présentations: Le corps enseignant au BasCanada: une féminisation réalisée, en cours ou à venir? Le cas de l'île de Montréal, présentée au congrès de l'Institut d'histoire de l'Amérique française tenu au Collège militaire royal de Saint-Jean-surRichelieu en octobre 1990 et Les maîtres et les maîtresses d'écoles lä̈ques indépendantes de l'île de Montréal, en 1825 et en 1835, présentée au Congrès biennal de l'Association canadienne d'histoire de l'éducation, tenu à l'Université d'Ottawa en octobre 1990.

16. Jacques Viger, Enseignement public dans le Comté de Montréal, en 1825 et Enseignement public dans le Comté de Montréal, en 1835, Archives du Séminaire de Québec, Fonds Verreau, manuscrits 018 et $018 \mathrm{a}$. On trouvera la description de ces sources et de leur contexte de réalisation dans Andrée Dufour, «Deux recensements manuscrits des écoles de l'île de Montréal en 1825 et en 1835: une contribution de Jacques Viger à l'histoire de l'éducation au Québec», Revue d'histoire de l'éducation/Historical Studies in Education, 5,3 (octobre 1988): 19-26 et Andrée Dufour, «Diversité institutionnelle et fréquentation scolaire dans l'île de Montréal en 1825 et 1835», Revue d'histoire de l'Amérique française, 41,4 (printemps 1988): 507-535.

17. La population résidant dans la cité s'élève à 22540 alors que la population rurale est de 14739 . 
viève, Sainte-Anne, Pointe-Claire, Lachine et dans les «divisions» de la paroisse de Montréal. Mais, même dans la partie rurale de l'île, les enseignantes étaient plus nombreuses que les enseignants. Viger y dénombra en effet 9 maîtres et 11 maîtresses en 1825. Et la féminisation se poursuivit puisque, en 1835, 12 maîtres et 19 maîtresses constituaient respectivement $39 \%$ et $61 \%$ du personnel enseignant des écoles rurales. Il serait toutefois imprudent d'étendre cette constatation à l'ensemble du Bas-Canada. Une enquête qui porte sur les années 1825 à 1860 et qui concerne surtout le milieu rural est nécessaire.

Les comptes publics publiés annuellement en appendice des Journaux de la Chambre d'Assemblée du Bas-Canada (JCABC) fournissent des indices précieux pour la connaissance du phénomène au moment des premières années d'existence du régime des écoles dites «de syndics» ou «d'Assemblée». On y trouve, de 1830 à 1832, la liste des salaires, des «allouances» comme on l'écrivait à l'époque, versés aux maîtres et aux maîtresses d'école des paroisses de campagne du Bas-Canada en vertu de la loi de 1829, ainsi que les noms et prénoms des personnes rémunérées. En ne retenant que les personnes dont le prénom ne laisse planer aucun doute sur leur genre, on arrive à un total de 510 maîtres pour les derniers six mois de l'année 1829, 277 hommes et 233 femmes, soit respectivement $54 \%$ et $46 \%{ }^{18}$. Comme le succès des écoles de syndics est toutefois particulièrement spectaculaire à partir de 1830, 1015 maîtres et maîtresses apparaissent dans les listes cette année-là. Par la même méthode, il est possible d'établir le genre de 923 de ces personnes, 488 hommes (53\%) et 435 femmes $(47 \%)^{19}$. Les listes salariales ne tiennent pas compte des 84 maîtres œuvrant dans les 82 écoles de l'Institution royale, des hommes pour la plupart, rétribués d'après la loi de l'Institution royale de 1801. La forte présence des femmes dans l'enseignement au Bas-Canada dès le tournant de la décennie 1830 est néanmoins perceptible. Cette présence se confirme et s'accentue en 1831. Ici, le «Tableau général de l'éducation» établi la même année par le Comité d'éducation de la Chambre d'assemblée ${ }^{20}$, à la suite des rapports effectués par les visiteurs d'école nouvellement nommés, a été utilisé ${ }^{21}$. Les visiteurs ont

\footnotetext{
18. JCABC 1830 (vol. 39), appendice $\mathrm{M}$ (comptes publics), $\mathrm{n}^{\mathrm{O}} 8$.

19. JCABC 1831 (vol. 40), appendice $\mathrm{P}$ (comptes publics), $\mathrm{n}^{\mathrm{O}} 8$.

20. Doté par la Chambre du pouvoir d'enquêter, d'interroger des témoins, de faire des rapports et recommandations relatives à l'éducation, le Comité d'enquête sur l'éducation devient permanent en 1831 et publie dès lors un, voire plusieurs rapports annuels.

21. Ce tableau est repris dans les $J C A B C$ de 1831-1832, Appendice I.I. On ne peut s'appuyer sur les comptes publics de 1831, car le scribe s'est contenté, dans $31 \%$ des cas, de mentionner les seules initiales des personnes rétribuées par l'État. D'ailleurs, à partir de 1832, les listes de maîtres et de maîtresses d'écoles subventionnées n'apparaissent plus dans les comptes publics.
} 
dénombré 1305 maîtres et maîtresses enseignant dans les 1216 écoles, tant publiques que privées du Bas-Canada. De ce nombre, 635 (49\%) sont des femmes. Celles-ci occupent désormais dans l'enseignement primaire une place aussi importante que celle de leurs collègues masculins.

Les différents rapports des Comités d'éducation parus dans les $J C A B C$ de 1832 à 1836 ne permettent malheureusement plus d'établir la proportion respective d'enseignants et d'enseignantes. Mais on peut raisonnablement avancer que les femmes sont plus nombreuses que les hommes dans l'enseignement primaire dès la première moitié de la décennie 1830 .

L'enquête de la commission Buller sur l'état de l'éducation au BasCanada tend à confirmer cette hypothèse. Cette importante enquête fut entreprise à la demande de lord Durham et menée de fait par Christopher Dunkin en 1838 et $1839^{22}$. Destinée à recueillir des renseignements de toutes sortes en vue de l'établissement de nouvelles structures éducatives, elle ne concerne qu'un nombre relativement restreint de collectivités (70), et touche très peu celles faisant partie de la grande région de Montréal ${ }^{23}$. Il demeure que l'examen des réponses fournies par 47 paroisses et townships ${ }^{24}$ fait apparaître une majorité d'enseignantes: 31 des 56 maîtres (sur un total de 61) dont on mentionne le prénom sont en effet des femmes.

Lors de l'établissement, en 1841, d'un nouveau système d'instruction public, un système largement inspiré d'ailleurs des recommandations contenues dans le rapport d'Arthur Buller, le surintendant Jean-Baptiste Meilleur se vit confier la tâche de faire rapport sur la situation des écoles du Bas-Canada. Débordé de travail et confronté à la fameuse «guerre des éteignoirs», il se limita essentiellement à des statistiques concernant le nombre d'écoles et d'élèves. Il faudra attendre la nomination des inspecteurs pour que les statistiques scolaires prennent, à partir de 1853, une importance sans précédent dans les rapports du surintendant et que les maîtres soient enfin comptabilisés. Ces statistiques, utilisées pour la créa-

22. L'enquête Buller est publiée dans le Rapport Durham et a été reprise par C. P. Lucas, Lord Durham Report on the Affairs of British North America (Oxford, Clarendon Press, 1912) III: app. D.

23. Ces questionnaires d'enquête sont reproduits dans les British Parliamentary Papers de 1839, $\mathrm{n}^{\circ}$ 2, App. D, 178-182. Quant aux réponses, elles sont conservées aux Archives nationales du Canada (ANC) dans le fonds RG4 B30. Ici, j'ai plus particulièrement consulté le questionnaire 2 de l'enquête, questionnaire qui concernait expressément les maîtres d'école. On trouvera une critique de cette source dans Allan Greer, «The Pattern of Literacy in Québec, 1745-1899», Histoire sociale/ Social History, 11,22 (novembre 1978).

24. J'ai dû écarter 23 questionnaires (ou tableaux) dont la fragilité du papier a rendu la consultation impossible. 
tion du tableau 1, permettent de constater un processus de féminisation déjà bien avancé du corps enseignant au début des années 1850 et son accentuation de 1853 à 1859 . Les causes de ce phénomène sont nombreuses ${ }^{25}$.

TABLEAU 1

RÉPARTITION DES INSTITUTEURS ET DES INSTITUTRICES DU BAS-CANADA DE 1853 À 1859

\begin{tabular}{lcccc} 
Année & \multicolumn{2}{c}{ Instituteurs } & \multicolumn{2}{c}{ Institutrices } \\
& nombre & $\%$ & nombre & $\%$ \\
1853 & 808 & 37 & 1404 & 63 \\
1854 & 925 & 38 & 1493 & 62 \\
1855 & 975 & 33 & 1812 & 67 \\
1856 & 892 & 32 & 1877 & 68 \\
1857 & 902 & 33 & 1850 & 67 \\
1858 & 972 & 33 & 1945 & 67 \\
1859 & 976 & 31 & 2129 & 69
\end{tabular}

Source: Journaux de l'Assemblée législative de la Province du Canada (JALPC) de 1854 à 1860.

\section{La question des salaires}

Examinons en premier lieu la question des salaires. Soucieuse d'assurer le succès de son système scolaire et de rehausser le prestige des maîtres par des salaires plus élevés, la Chambre d'assemblée avait prévu, dans la loi de 1829, le paiement du salaire du maître ou de la maîtresse d'école, un salaire fixé à $20 £$ par an. Cette disposition n'était pas nouvelle puisque les maîtres de l'Institution royale recevaient le même traitement depuis 1801. Mais l'État se faisait plus généreux à l'égard des autres maîtres des campagnes. En plus de l'allocation de base, il leur versait une compensation annuelle de dix shillings par enfant instruit gratuitement, pourvu que le nombre de ces écoliers ne fut pas inférieur à 20 ni supérieur à 50 . Cette innovation, qui avait pour but d'attirer les enfants pauvres dans les écoles, garantissait déjà aux maîtres un revenu plus substantiel qui pouvait aller jusqu'à $45 £$ par

25. Ces causes et leurs fondements documentaires ont été partiellement évoqués dans ma thèse de doctorat, op. cit., publiée sous le titre Tous à l'école. État, communautés rurales et scolarisation au Québec de 1826 à 1859 (Montréal, Hurtubise HMH, 1996). 
$\mathrm{an}^{26}$. À cela s'ajoutait la traditionnelle rétribution que versaient mensuellement aux maîtres les parents en mesure de payer pour l'instruction de leurs enfants. Le montant de la rétribution n'était pas précisé en 1829, mais pouvait s'élever sans doute jusqu'à trois shillings et quatre deniers par écolier, tel que le stipulaient les règlements accompagnant la loi de 1801. Grâce à ces trois sources de paiement, le travail des mâ̂tres s'avérait plus rentable et assurément plus valorisé, attirant tant les hommes que les femmes dans les écoles élémentaires publiques.

En 1832, le législateur porta même l'allocation annuelle de base à $24 £$. Désireux cependant de réduire les dépenses pour l'éducation, plus élevées que prévu en raison du succès grandissant des écoles de syndics, il limita à une le nombre d'écoles par arrondissement scolaire, restreignit à dix le nombre d'enfants instruits gratuitement dans chaque école et fixa la rétribution mensuelle à deux shillings par enfant. Les revenus des maîtres pouvaient en être sensiblement diminués. Aussi, plusieurs maîtres quittèrent l'enseignement et autant d'écoles fermèrent leurs portes, comme l'écrivit le curé F.-X. Delage de l'Islet:

Ces différentes écoles [de syndics] ont commencé dans l'automne de 1829 et ont continué jusqu'au printemps 1832, époque où le Bill de 1832 a commencé à être mis à exécution; ce qui a occasionné la cessation de plusieurs écoles, vu que le nombre d'arrondissements avait été diminué et que l'allocation des instituteurs était devenue moindre ${ }^{27}$.

Des habitants du comté de Kamouraska déplorèrent amèrement le départ des instituteurs. Ces dispositions nouvelles, ces «monstrueuses imperfections» de la loi, écrivirent-ils au gouverneur, ont fait que «les instituteurs effrayés ont fuit [sic] ou se disposent à fuir l'esclavage qu'on leur offre, et le morceau de pain de l'indigence ${ }^{28}$ ». Lorsque la loi scolaire du 3 avril 1833 ramena l'allocation gouvernementale à $20 £$, le salaire d'avant 1832 , en offrant une prime de $4 £$ seulement à ceux et celles capables d'enseigner l'anglais, on peut penser que les instituteurs quittèrent encore davantage le métier.

Le mode de financement du nouveau système d'instruction public établi en 1841 différait beaucoup de celui prévalant durant les années 1829 à 1836. Largement inspiré des recommandations contenues dans le

26. Ce revenu était supérieur à celui des domestiques qui recevaient de 16 à $22 £$ par an, Andrée Dufour, «Diversité institutionnelle...», 531.

27. Lettre du 3 janvier 1833 conservée aux ANC, Fonds RG4 B30, vol. 109.

28. Lettre reprise dans les JCABC de 1832-1833, 270. 
rapport Buller, il reposait non plus essentiellement sur la générosité de l'État, mais sur une participation aussi importante des collectivités locales par le moyen d'une taxe foncière. L'allocation que versait directement l'État aux maîtres et aux maîtresses disparaissait. La loi laissait désormais aux commissaires d'école le soin de fixer le pourcentage des subventions gouvernementales et des taxes scolaires locales accordé aux enseignants. La rétribution mensuelle était maintenue mais ramenée à 1 shilling par mois. Quant à l'indemnité de 10 shillings accordée pour les enfants instruits gratuitement, elle était abolie. On ne peut l'affirmer, faute de données pour les années 1840, mais ces dispositions, qui signifiaient le retrait de l'État du paiement des maîtres et maîtresses d'école, contribuèrent sans doute à tenir les hommes éloignés des salles de classe et à la féminisation du personnel enseignant.

Pour sa part, André Labarrère-Paulé attribue en grande partie l'importance grandissante des femmes dans le métier à l'avarice des commissaires d'école qui versaient des bas salaires aux institutrices et à l'acceptation de tels salaires par des jeunes filles passives, inexpérimentées et incompétentes ${ }^{29}$. Certes, à la suite de la disparition de l'allocation directe de l'État, une allocation identique pour les maîtres et les maîtresses d'école, rappelons-le, la moyenne des salaires que recevaient les institutrices était devenue généralement inférieure à celle des instituteurs et on engageait les premières de préférence aux seconds. Mais bien d'autres raisons que celles avancées par Labarrère-Paulé expliquent ces pratiques.

La perception du travail des femmes comme une source de revenus d'appoint ou temporaire était sans doute présente à l'esprit des commissaires. La pauvreté de certaines communautés rurales, en particulier des établissements nouveaux caractérisés par une population peu nombreuse et dispersée, était souvent la cause de l'engagement de femmes à qui l'on versait de plus maigres émoluments ${ }^{30}$. En ajoutant à cela la faiblesse des subventions gouvernementales octroyées à ces communautés, de même que le peu d'empressement de certains parents ou propriétaires, peu sensibles à l'instruction et au rôle des maîtres, à verser leurs contributions ${ }^{31}$ ainsi que la crainte ou l'incapacité des commissaires de poursuivre en jus-

29. Dans Les instituteurs lä̈ques..., André Labarrère-Paulé revient sans cesse sur cette assertion. Voir, notamment, les pages 93,133,142,179 et 299.

30. Voir les rapports pour l'année 1852 des inspecteurs Georges Tanguay, Césaire Germain et John Bruce (JALPC 1853, App. J.J.). Voir également la lettre du 8 janvier 1849 des dissidents du township de Farnham (ANQ, Fonds E13, correspondance reçue de 1848, n $1347^{1} / 2$ ).

31. Voir le rapport de 1852 de Peter Winter, inspecteur des comtés de Gaspé et de Bonaventure (JALPC 1853, App. J.J.) et celui de l'inspecteur Pierre-M. Bardy pour l'année 1853 (JALPC 1854-1855, App. B). 
tice pour recouvrer les arrérages des différentes cotisations ${ }^{32}$, on explique autrement les maigres salaires versés, notamment aux femmes. Certains habitants agissaient de bonne foi, tels ceux d'une municipalité reculée du comté de Deux-Montagnes qui croyaient que 16 livres constituait un bon salaire pour un instituteur ${ }^{33}$. Des commissaires engageaient les instituteurs qui demandaient les salaires les plus bas, croyant bien faire en économisant ainsi les taxes perçues ${ }^{34}$. Notons cependant que plusieurs collectivités, souvent les plus anciennes, savaient apprécier l'importance d'instituteurs et d'institutrices compétents et leur offraient des salaires relativement élevés. À Percé, par exemple, on était prêt à débourser $75 £$ par an pour trouver un bon instituteur ${ }^{35}$. Mais c'était insuffisant, semblet-il, pour garder les hommes dans les salles de classe. Durant la décennie 1850 , des emplois nettement plus lucratifs que l'enseignement attiraient les jeunes hommes, comme l'écrivait l'inspecteur John Hume: «Le prix extraordinaire des gages qui se sont donnés récemment dans toutes les sortes d'emplois a porté un grand nombre de personnes qui jusque-là s'étaient livrées à l'enseignement, à abandonner cet emploi pour en embrasser d'autres qui étaient plus rétributifs $[\text { sic }]^{36}$.» L'inspecteur John Roney écrivit dans son rapport de janvier 1856: «peu de jeunes gens choisissent l'enseignement comme profession permanente; ils regardent cet état comme un marche-pied [sic] qui doit les faire parvenir à quelque chose de plus lucratif et l'abandonnent aussitôt que l'occasion s'en présente $^{37} . »$

En définitive, la diminution des salaires versés aux enseignants et, surtout, aux enseignantes, joua un rôle important dans la féminisation du corps enseignant qui survint au Bas-Canada dès les années 1830 et qui s'accentua au cours des décennies suivantes. Les motifs qui en furent à l'origine ne peuvent toutefois être réduits à l'avarice des commissaires ou à la passivité des jeunes maîtresses d'école.

32. Voir le rapport pour l'année 1854 de l'inspecteur Magloire Lanctôt et celui de l'inspecteur Bardy pour 1853 (JALPC 1854-1855, App. B).

33. Ce cas est rapporté par l'inspecteur John Bruce après sa visite du township de Grenville, faite en 1852 (JALPC 1853, App. J.J.). App. B).

34. Voir le rapport de l'inspecteur Cléophe Cimon pour l'année 1854 (JALPC 1854-1855,

35. Voir le rapport de l'inspecteur Georges Tanguay du 25 novembre 1852 (JALPC 1853, App. J.J.).

36. Second rapport pour l'année 1854 (JALPC 1854-1855, App. B); voir aussi le rapport de l'inspecteur Parmelee pour l'année 1855 (JALPC 1856, App. 16).

37. JALPC 1856, App. 16. Voir également, dans les mêmes $J A L P C$, le rapport de l'inspecteur Georges Tanguay pour 1855. Il est difficile d'estimer la nature et les salaires de «toutes [ces] sortes d'emplois» «plus rétributifs». Le clerc du surintendant Meilleur recevait cependant $60 £$ par an. 


\section{L'intérêt divergent des hommes et des femmes pour l'enseignement primaire}

Les femmes bas-canadiennes un tant soit peu instruites trouvèrent en effet dans l'enseignement l'une des rares occasions qui leur étaient offertes de gagner leur vie. Elles percevaient également le métier d'institutrice comme une excellente préparation à leur futur rôle de mère ${ }^{38}$. Il apparaît également que plusieurs femmes furent aussi désireuses de mettre avantageusement à profit des connaissances relativement étendues acquises dans les établissements du Bas-Canada ou à l'étranger. J'y reviendrai.

Les perspectives masculines étaient tout autres. Comme on l'a vu plus haut, les hommes se retirèrent des écoles rurales lorsqu'ils se virent offrir, dans la fonction publique notamment, des salaires supérieurs aux traitements consentis dans l'enseignement primaire. Des traitements diminués qui ne permettaient sans doute plus de supporter une famille. Les hommes instruits répugnaient également à faire carrière au niveau primaire. Aussi, malgré bien des efforts, les surintendants ne parvinrent pas, on le verra plus loin, à les y attirer en plus grand nombre.

Les historiennes Marta Danylewycz, Beth Light et Alison Prentice ont montré que la féminisation de l'enseignement tenait notamment des diverses possibilités de travail existant alors pour les hommes en milieu rural. Ainsi, dans certaines régions rurales du Québec, tels les comtés de Montmagny et de Terrebonne qu'elles ont étudiés, des activités comme le travail en forêt et la pêche attiraient davantage les hommes, occasionnant la diminution des effectifs masculins dans l'enseignement et le renforcement de la présence traditionnelle des femmes ${ }^{39}$.

Les femmes remplacèrent également plusieurs instituteurs congédiés pour inconduite ou absentéisme. Et alors que les instituteurs, à l'instar des enseignants religieux, préféraient enseigner dans les localités facilement accessibles et bien établies ${ }^{40}$, elles consentirent à tenir école dans les parties les plus isolées des comtés et dans les nouvelles régions de colonisation. Les inspecteurs devaient d'ailleurs souligner en maintes occasions la contribution de jeunes institutrices à la diffusion de l'instruction dans les régions périphériques ${ }^{41}$.

38. Marta Danylewycz, Beth Light et Alison Prentice, «The Evolution...», 82.

39. Ibid., 104.

40. C'est ce qu'ont soutenu Marta Danylewycz, Beth Light et Alison Prentice, Ibid., 105.

41. Voir, entre autres, le rapport de l'inspecteur J.N.A. Archambault du 25 août 1852 (JALPC 1853, App. J.J.) et celui de l'inspecteur Georges Tanguay pour l'année 1856 (JALPC 1857, App. 58). 


\section{L'affirmation de l'Église catholique}

La venue de l'État dans l'éducation, concrétisée par les lois de l'Institution royale (1801) et de l'Assemblée (1829), l'engagement de la bourgeoisie à travers les sociétés d'éducation ainsi que l'existence de nombreuses écoles indépendantes ${ }^{42}$ avaient considérablement réduit l'importance de l'Église dans l'enseignement primaire durant les premières décennies du XIX ${ }^{\mathrm{e}}$ siècle. Comme on le sait, les jésuites et les récollets avaient disparu et les sulpiciens concentraient leurs activités éducatives dans la paroisse de Montréal où ils tenaient le collège et supportaient de petites écoles confiées aux religieuses de la Congrégation de Notre-Dame et à des laïques. Les frères des Écoles chrétiennes n'arrivèrent qu'en 1837. Du côté des religieuses, seules les dames de la Congrégation, les ursulines et les sœurs de l'Hôpital Général enseignaient à quelques centaines d'élèves dans une vingtaine de pensionnats/écoles répartis, en 1825, dans les régions de Montréal, Québec et TroisRivières ${ }^{43}$. Comme le montre le tableau 2, on observe même une diminution de l'importance relative des enseignants religieux et cela, à la faveur de l'établissement des écoles de syndics où l'on engage des enseignants laïques, des femmes en majorité

Mais l'affirmation de l'Église à partir de 1840, sous l'impulsion de l'énergique évêque Ignace Bourget, contribua à la féminisation du métier, par la croissance de ses effectifs en premier lieu. Rappelons brièvement

TABLEAU 2

RÉPARTITION DU PERSONNEL ENSEIGNANT DE LA PARTIE RURALE DE L'ÎLE DE MONTRÉAL EN 1825 ET 1835, SELON LE STATUT ET LE SEXE

$\begin{array}{lcccccc} & & 1825 & & & 1835 & \\ \text { Religieux } & \text { Hommes } & \text { Femmes } & \text { Total } & \text { Hommes } & \text { Femmes } & \text { Total } \\ \text { Laïques } & 1 & 7 & 8 & 5 & 4 & 9 \\ \text { Total } & 8 & 4 & 12 & 11 & 15 & 26 \\ & 9 & 11 & 20 & 16 & 19 & 35\end{array}$

Source: Jacques Viger, Enseignement public..., en 1825 et Enseignement public..., en 1835 (ASQ, Fonds Verreau, manuscrits 018 et $018 \mathrm{a}$ ).

42. Pour mesurer l'importance des sociétés d'éducation et des écoles indépendantes, voir Andrée Dufour, «Diversité institutionnelle...», 507-535.

43. Micheline Dumont, L'instruction des filles au Québec (1639-1960) (Ottawa, Société historique du Canada, coll. «Brochures historiques, $\left.n^{0} 49,1990\right), 11$. 
que, de 1842 à 1860, dix nouvelles congrégations enseignantes de femmes furent fondées ou s'installèrent au Québec et que, durant la même période, trois congrégations enseignantes masculines s'implantèrent ou se réimplantèrent - ce fut le cas des jésuites en 1842 - ici. Si bien que le pourcentage de religieux chez les enseignants passa de $4 \%$ au Québec en 1843-1844 à plus de 10\% en 1853. La proportion des enseignantes et enseignants religieux augmentera sensiblement par la suite, mais plus rapidement pour les femmes que pour les hommes. Ainsi, en 1867-1868, $11,2 \%$ des instituteurs sont religieux, alors que la proportion de religieuses chez les institutrices est de $22,1 \%$, soit quasi le double. Il ne faudrait toutefois pas exagérer la portée de cette cléricalisation, en milieu rural du moins. En effet, de 1840 à 1867, les religieuses se cantonnèrent presque exclusivement dans les villes, particulièrement celles de la région montréalaise ${ }^{44}$.

Par ailleurs, la fréquente opposition des membres du clergé à l'engagement d'instituteurs pour enseigner dans les écoles mixtes - elles étaient la norme en milieu rural - contribua à l'augmentation du nombre d'institutrices ${ }^{45}$, d'autant que les filles, qui fréquentaient nettement moins l'école que les garçons durant la première moitié du $\mathrm{XIX}^{\mathrm{e}}$ siècle $^{46}$ vont effectuer un rattrapage à ce chapitre durant la seconde moitié du siècle. On attribue ce rattrapage à une volonté accrue d'instruire les filles à l'école et au retrait plus précoce de l'école des garçons, que les parents envoyaient travailler aux champs ou en forêt ${ }^{47}$.

\section{La préférence marquée des collectivités locales}

Ce fut aussi, dans de nombreux cas, pour leur compétence, leur dévouement, leur aptitude à l'enseignement et leur attention à l'égard des enfants que les institutrices furent préférées aux instituteurs par les collectivités rurales. Ainsi, dans son rapport d'avril 1854, l'inspecteur John Bruce, longtemps maître d'école réputé de Montréal, écrit:

[...] la discipline dans les écoles tenues par les femmes est généralement plus observée; elles paraissent porter plus d'attention à la santé, au bien-être et à la propreté de leurs élèves, et en général elles sont plus disposées à marcher dans la voie du progrès, à

44. Bernard Denault et Benoît Lévesque, Élements pour une sociologie des communautés religieuses au Québec (Montréal, Les Presses de l'Université de Montréal, 1975), 74-86.

45. L'évêque Lartigue notamment, qui menaçait cette pratique du refus des sacrements, et plusieurs curés. Au sujet de ceux-ci, voir le rapport de l'inspecteur Pétrus Hubert pour 1854 (JALPC 1854-1855, App. B).

46. Allan Greer, loc. cit., 321 et Andrée Dufour, «Diversité institutionnelle...», 523.

47. Marta Danylewycz, Beth Light et Alison Prentice, «The Evolution...», 91. 
améliorer leurs systèmes d'enseignement que les instituteurs. Je trouve aussi que leur méthode pour perfectionner les élèves ne le cède en rien à celui $[s i c]$ des instituteurs ${ }^{48}$.

Jean Crépault, un ancien instituteur devenu inspecteur, invoqua des raisons similaires pour expliquer au surintendant l'attachement des gens de l'île aux Grues à leur maîtresse d'école: «Cette demoiselle a fait faire des progrès rapides à ses élèves et les a disciplinés comme il est rare de voir. Aussi est-elle en vénération chez le peuple qui a pour elle les considérations les plus marquées ${ }^{49}$.»

Devant la préférence des commissaires, des parents et même de ses proches collaborateurs pour les institutrices, le surintendant Meilleur, qui pourtant voulait faire de l'enseignement un métier masculin, devait convenir d'une certaine supériorité des femmes:

[...] on les préfère généralement aux hommes, surtout pour tenir les écoles fréquentées par de jeunes enfants, parce qu'elles savent mieux condescendre à la faiblesse de leur âge, la pratique des moyens propres à s'insinuer dans leur estime, et à gagner leur confiance et leur affection.

La propreté, le bon ordre, une douce discipline et un progrès signalé caractérisent généralement les écoles tenues par les femmes $[\ldots]^{50}$.

Cette admission ne devait pas empêcher le surintendant de tout mettre en œuvre pour favoriser les instituteurs. Dans cet esprit, il suggéra la mise sur pied de bureaux d'examinateurs qui auraient pour effet

de donner à l'enseignement le caractère et la permanence d'une profession, d'un état fixe, offrant à ses membres une garantie des considérations et des ressources dont ils ont besoin. Cette garantie, en encourageant les Instituteurs à demeurer chacun à son poste, nous assurerait de leur activité, de leur constance, et de leur dévouement dans la tâche qui leur est imposée. Cette garantie des considérations et des ressources convenables dans l'enseignement servirait encore d'appas puissant à nos jeunes gens instruits, qui, au sortir de nos collèges et de nos lycées, cherchent, en dehors de professions déjà trop remplies, un genre d'occupations analogues à leur éducation, à leur goût, à leur vocation ${ }^{51}$.

48. Rapport d'avril 1854 (JALPC 1854-1855, App. B)

49. Rapport de juillet 1852 (JALPC 1853, App. J.J.).

50. Rapport du 11 mai 1854 (JALPC 1854-1855, App. B).

51. Rapport du 15 avril 1846 (JALPC 1846, App. P). 
Il devait en outre recommander à plusieurs reprises que l'on verse aux instituteurs une rémunération convenable, afin de reconnaître leur compétence et leur mérite, mais aussi pour s'assurer d'une «coopération», d'une présence plus importante des hommes dans l'enseignement ${ }^{52}$. En 1848, il suggéra aussi d'établir une école normale pour accueillir les jeunes hommes issus des collèges et des lycées qui préféraient enseigner dans les écoles modèles ${ }^{53}$. Parallèlement, il proposa à l'exécutif de lui accorder les fonds nécessaires à l'octroi de bourses pour permettre à des instituteurs de campagne et à d'autres jeunes gens prometteurs de «venir dans les villes prendre des leçons sur certaines branches d'enseignement dans nos écoles supérieures ${ }^{54} \gg$.

Pour faciliter aux instituteurs ruraux l'accès au brevet d'enseignement, Meilleur recommanda dans son rapport du 20 avril 1853 l'ouverture de bureaux d'examinateurs dans chacun des districts judiciaires ${ }^{55}$. Il avait cru qu'en obligeant les seuls hommes à passer l'examen du bureau d'examinateurs, les communautés rurales n'engageraient dorénavant que des instituteurs brevetés. Rien n'y fit. Les commissaires continuèrent d'engager des femmes ou des jeunes filles. Et cela, comme nous l'avons vu plus haut, pour des raisons d'économie mais aussi pour bien des motifs qui dépassaient le simple «arbitraire de commissaires d'écoles ignorants et parcimonieux ${ }^{56}$ ». Il se résigna donc à recommander, en 1854, que l'on formât à l'école normale tant les institutrices que les instituteurs ${ }^{57}$.

\section{L'attitude du surintendant Chauveau}

Son successeur, Pierre Chauveau, devait lui aussi constater la diminution du nombre d'instituteurs et l'augmentation considérable de celui des institutrices. En 1856, celles-ci constituaient désormais 67\% du corps enseignant (voir le tableau 1). Pour renverser la tendance, Chauveau fit part de son intention de recruter les futurs normaliens non plus parmi les finissants des collèges classiques, trop attirés par les professions libérales, selon lui, mais parmi ceux qui quittaient les études après la classe de Belles-Lettres ou de Rhétorique ou après l'école modèle ${ }^{58}$. Pensant peut-être que les institutrices répugneraient à passer l'examen des bureaux d'examinateurs, il

52. Notamment dans son rapport du 12 juin 1851 (JALPC 1853, App. J.J.).

53. Rapport du 11 mars 1848 (JALPC 1848, App. P).

54. Rapport du 11 mars 1848 (JALPC 1848, App. P).

55. JALPC 1853, App. J.J.

56. La citation est tirée du mémoire adressé par Jacques Crémazie au Comité d'enquête Sicotte en avril 1853 (JALPC 1853, App. J.J.).

57. Rapport du 11 mai 1854 (JALPC 1854-1855, App. B).

58. Rapport du 28 février 1856 (JALPC 1856, App. 16). 
suggéra d'étendre aux institutrices l'obligation de détenir un brevet. «C'est en grande partie la concurrence des institutrices sans diplômes, qui ruine les instituteurs et favorise l'avarice de quelques municipalités», soutint-il ${ }^{59}$. La loi scolaire de 1846 avait accordé dix ans aux instituteurs pour obtenir leur brevet. Le législateur avait ensuite, en 1849, ramené l'échéance à 1852, un délai encore lointain. Aux institutrices, la loi de 1856 n'accorda qu'un répit d'un an pour se soumettre à l'examen. Ce court sursis ne diminua pas leur ardeur. Dès l'année suivante, le pourcentage d'institutrices détentrices d'un brevet rejoignait celui des instituteurs. Je reviendrai plus loin sur ce résultat sans doute quelque peu étonnant pour les autorités scolaires.

Dans sa circulaire du 21 juillet 1856 adressée aux commissaires et syndics dissidents, Chauveau fit volte-face. Il manifesta le désir de les voir augmenter la cotisation de manière à pouvoir verser aux instituteurs et aux institutrices des salaires respectifs d'au moins $50 £$ et $25 £$. À première vue, la recommandation peut sembler louable puisqu'elle fixait un salaire minimum et signifiait une hausse de revenus pour bon nombre de maîtres et de maîtresses. Mais Chauveau suggérait un important écart de salaire entre les deux sexes, ce qui faisait plus que reconnaître implicitement une pratique existant depuis 1842 pour tout un ensemble de motifs. Il l'entérinait en quelque sorte. Comment, après cela, reprocher aux commissaires, leur «avarice», la «ruine des instituteurs» et, en définitive, leur imputer la responsabilité première d'une «catastrophique» féminisation de l'enseignement?

\section{LES MAÎTRESSES D'ÉCOLE}

Cette féminisation fut-elle vraiment une catastrophe? Ces institutrices rurales de plus en plus présentes dans les écoles primaires étaientelles tellement trop jeunes, aussi inexpérimentées et aussi incompétentes et passives que le prétend André Labarrère-Paulé?

\section{Pas si jeunes et si inexpérimentées}

Examinons d'abord leur âge et leur expérience pédagogique. C'est dans le rapport du Comité d'enquête Sicotte qu'on trouve les premières statistiques concernant l'âge des enseignantes. Constitué en février 1853 par l'Assemblée législative du Canada-Uni pour enquêter sur «l'état de l'instruction primaire dans le Bas-Canada», le Comité avait fait parvenir une série de questions à tous les curés et ministres du culte ainsi qu'à tous les secrétaires-trésoriers des municipalités scolaires. Il avait aussi dépouillé les premiers rapports des inspecteurs. Son rapport déposé en juin 1853 faisait,

59. Rapport du 28 février 1856 (JALPC 1856, App. 16). 
entre autres, état de l'âge de 822 institutrices, qu'il répartissait dans trois groupes d'âge: 118 avaient de 15 à 17 ans, 193 de 17 à 19 ans et 343 étaient âgées de 20 à 24 ans.

On remarquera que le Comité avait omis d'indiquer le nombre d'institutrices âgées de 25 ans ou plus. Elles étaient 168. Ces quatre catégories d'âge représentaient respectivement $14 \%, 23 \%, 42 \%$ et $20 \%$ des enseignantes. Certes, 15, 16 et 17 ans peuvent nous sembler des âges bien tendres pour enseigner, même si la grande majorité des élèves avaient moins de 12 ans. Ces jeunes filles constituaient cependant moins de $15 \%$ de la totalité des institutrices, celles ayant 20 ans et plus formant l'importante majorité. L'inspecteur J. N. A. Archambault corrigeait d'ailleurs, après une première visite de son district, l'impression répandue d'institutrices trop jeunes: «Je dis [...] que c'est manquer à la vérité, de prôner que ces institutrices pour la plupart n'ont pas atteint la quinzième année. Dans les trois comtés que je parcours, je n'en ai trouvé que deux au-dessous de l'age [sic] de dix-sept ans ${ }^{60} . \gg$ Des institutrices des écoles primaires des comtés de Chambly, Verchères et Richelieu, il écrivait également:

Il faut entrer dans ces écoles comme nous le faisons nous dans nos parcours, à l'improviste, pour voir la bonne tenue de ces écoles. J'en ai été souvent frappé, car, le plus souvent, nous arrivons sans être attendus; et toujours vous trouvez ces demoiselles en bonne tenue, les classes en bon ordre, les enfants sages et bien disciplinés, leurs livres et papiers en bonne condition ${ }^{61}$.

Le jeune âge n'était pas nécessairement signe d'inexpérience et d'incompétence. Il était en effet fréquent que les maîtresses âgées de 25 ans ou plus et même bon nombre de celles âgées de 20 à 24 ans comptent déjà plusieurs années d'expérience. Le rigoureux inspecteur John Bruce ${ }^{62}$ signalait, par exemple, le cas d'une institutrice de 25 ans qui enseignait depuis dix ans et qu'il estimait par ailleurs très qualifiée pour tenir l'école modèle. Nous avons même retrouvé dans la correspondance adressée au surintendant en 1859 la trace d'institutrices, les sœurs Vénérande, Marianne et Marie Pellerin, qui enseignaient trente ans plus tôt dans les écoles de syndics $^{63}$. En octobre 1854, l'inspecteur et ancien instituteur Pierre F.

60. Rapport du 31 mars 1853 (JALPC 1853, App. 16).

61. Ibid.

62. L'inspecteur Bruce produisit de 1852 à 1866 des rapports volumineux et fort documentés et ce, de façon très régulière. Voir l'appréciation que fait de cet inspecteur Louis-Philippe Audet dans le Dictionnaire biographique du Canada, IX: De 1861 à 1870 (Québec, Les Presses de l'Université Laval, 1972), 102-103.

63. ANC, Fonds RG4 A1, vol. 284, lettre du 17 avril 1829 et ANQ, Fonds E13, correspondance reçue de 1859 , lettre $\mathrm{n}^{\mathrm{O}} 1137$. 
Béland écrivit à propos des institutrices de son district: «Un quart d'entre elles n'a pas l'âge requis ${ }^{64}$; et pourtant elles sont les plus qualifiées ${ }^{65}$.» Par ailleurs, l'inspecteur John J. Roney avait rencontré dans son district d'inspection, l'Outaouais, des écoles dirigées par des jeunes filles dont «l'aptitude à l'enseignement était supérieure à celles de la majorité des instituteurs ${ }^{66} \gg$.

Dans son rapport pour l'année $1859^{67}$, le surintendant Chauveau publiait par ailleurs la liste des instituteurs et institutrices inscrits au registre de la caisse d'épargne qu'il avait mise sur pied quelques années plus tôt. Parmi les 225 inscrits, nous avons pu identifier par leur prénom 115 instituteurs et 84 institutrices. Il leur était possible de souscrire à la caisse en fonction de leurs années d'expérience dans l'enseignement et ce, jusqu'à un maximum de dix ans. Les instituteurs avaient cotisé pour 8,4 années, les institutrices pour 7,7. En définitive, on ne peut conclure à une infériorité significative des institutrices sur le plan de l'expérience et même de la compétence. Examinons cependant de plus près les qualifications de celles-ci.

\section{Des «incompétentes» assez souvent compétentes}

$\mathrm{Au}$ concept de compétence, je donne ici la définition suivante: connaissances et aptitudes reconnues pour accomplir une tâche donnée, par exemple enseigner, assurer la transmission des connaissances requises par la loi de l'éducation. Plus concrètement, être compétent(e) ou qualifié(e) pour l'enseignement élémentaire, cela signifiait, de 1801 à 1845, avoir la capacité de montrer à lire, à écrire et à compter ${ }^{68}$. Les institutrices rurales avaient-elles cette compétence? Le recensement scolaire de 1825 de Jacques Viger révèle que deux des trois maitresses laïques de la partie rurale de l'île de Montréal correspondaient à cette définition, l'une des trois n'enseignant que la lecture et l'écriture. Ce qui ne traduisait pas nécessairement chez elle une incapacité d'enseigner à compter. Lorsque les élèves étaient très jeunes, les enseignants se limitaient souvent à la lecture et à l'écriture, voire à la seule lecture ${ }^{69}$. Dix ans plus tard, 13 des 15 maîtresses laïques travaillant dans les 12 écoles recensées par Viger (trois de syndics, cinq indépendantes et quatre soutenues par les sulpiciens) dans la partie

64. Cet âge était de 18 ans selon les normes des bureaux d'examinateurs.

65. Rapport du 2 octobre 1854 (JALPC 1854-1855, App. B.).

66. Rapport du 19 mars 1853 (JALPC 1853, App. J.J.).

67. Publié dans les JALPC de 1860, App. 50.

68. Ces exigences étaient précisées dans les règlements et les documents accompagnant les lois de l'Institution royale et de syndics.

69. Nous avons constaté cette pratique dans les commentaires des notables justifiant leurs réponses aux questions de l'enquête Buller. 
rurale de l'île avaient déclaré au moins montrer la lecture, l'écriture et le calcul. Huit d'entre elles enseignaient en outre la grammaire, deux la géographie et une le latin... à des filles. Les maîtresses outrepassaient donc assez souvent les conditions minimales requises par la loi.

Une grande enquête, menée par le Comité d'éducation de la Chambre en novembre 1835 auprès de 48 des 77 députés élus dans 32 des 40 comtés du Bas-Canada, se révèle cependant plus représentative de la situation prévalant dans l'ensemble des campagnes de la colonie. Le comité avait interrogé ces députés responsables des écoles de syndics pour savoir s'il y avait, à leur connaissance, dans leur comté, des maîtres «incapables d'enseigner à lire, à écrire ou à compter dans la langue de la majorité». Sept députés reconnurent ne rien savoir à ce chapitre, étant élus depuis peu ou n'ayant pas visité les écoles de leur comté. Dix déclarèrent que, selon eux, les maîtres de leur comté n'étaient pas, peu ou assez peu qualifiés. Sept autres répondirent que la compétence variait selon les maîtres ou les matières enseignées et, enfin, le plus grand nombre, soit 24, affirmèrent que les mâ̂tres et les maîtresses étaient qualifiés ou même très qualifiés aux yeux de la $\operatorname{loi}^{70}$. On n'aurait donc retenu que les témoignages les plus accablants?

Les témoignages des députés ne permettent pas cependant de distinguer les instituteurs et les institutrices. L'enquête Buller de 1838-1839 comble cette lacune. D'après les réponses fournies par les notables interrogés (curés, ministres du culte, notaires, médecins, maîtres de poste, juges de paix, officiers de milice et écuyers), nous avons également réparti les 24 maîtres et 31 maîtresses d'école dénombrés selon le degré de leurs qualifications. Le tableau 3 rend compte de cette répartition et montre que la très

TABLEAU 3

\section{RÉPARTITION DES MAÎTRES ET DES MAÎTRESSES DE 47 LOCALITÉS RURALES DU BAS-CANADA, EN 1838, SELON LE DEGRÉ DE LEURS QUALIFICATIONS}

Possédant des qualifications jugées

Les meilleures, excellentes, très bonnes

Bonnes, relativement bonnes, suffisantes

Moyennes, passables

Insuffisantes, faibles, très faibles

$\begin{array}{cc}\text { Maîtres } & \text { Maîtresses } \\ 2 & 3 \\ 21 & 25 \\ 1 & 2 \\ 0 & 1\end{array}$

Source: Questionnaire 2 de l'enquête Buller (ANC, RG4 B30, vol. 108-115). 
grande majorité des maîtres et des maitresses qui étaient restés dans l'enseignement, malgré le non-renouvellement de la loi des syndics en 1836 et l'arrêt des subventions gouvernementales, possédaient les compétences requises pour enseigner dans les écoles élémentaires publiques.

La mention des matières enseignées permet en outre de constater qu'ils et qu'elles débordaient souvent le simple enseignement de la lecture, de l'écriture et du calcul. La grammaire, la géographie, l'histoire et aussi le latin étaient enseignés aux élèves. Sarah Farrand enseignait même la philosophie et la chimie, pas à tous ses élèves bien sûr, certains étant parfois bien jeunes. D'ailleurs l'écriture, dont l'apprentissage était alors considéré bien distinct de la lecture ${ }^{71}$, n'était montrée qu'à environ la moitié des élèves. Ceux-ci étant souvent débutants, seuls quelques-uns apprenaient l'arithmétique, nous révèlent les réponses de l'enquête.

Organisateur de l'enquête, Christopher Dunkin avait également demandé qu'on lui précise «où et comment les maîtres avaient été éduqués». L'examen des questionnaires retenus permet cette répartition pour les 31 maîtresses dénombrées:

TABLEAU 4

RÉPARTITION DES INSTITUTRICES DE 47 LOCALITÉS RURALES DU BAS-CANADA, EN 1838, SELON LE LIEU DE LEUR FORMATION

Lieu de formation des institutrices

Nombre

Dans un école de paroisse ou de canton

Au couvent (de la CND ou des Ursulines)

Dans une académie (du Bas-Canada ou des États-Unis)

Dans la famille

Au Canada

Au Canada et en Angleterre

En Irlande et en Écosse

Aux États-Unis

À Québec

1

Non indiqué

Source: Questionnaire 2 de l'enquête Buller (ANC, RG4 B30, vol. 108-115).

71. François Furet et Jacques Ozouf ont, dans Lire et écrire. L'alphabétisation des Français de Calvin à Jules Ferry (Paris, Éditions de Minuit, 1977), tome 1, bien montré la perception différente que l'on avait de ces deux apprentissages au $\mathrm{XIX}^{\mathrm{e}}$ siècle. 
Convenons que les réponses fournies manquent souvent de précision. On peut toutefois supposer chez plusieurs maîtresses, particulièrement chez celles instruites au couvent ou dans les académies, des connaissances supérieures à celles qu'exigeait la loi. Encore ici, il nous apparaît incorrect de traiter la plupart des maîtresses d'école d'incompétentes, de peu ou de mal qualifiées. Ajoutons que les premières écoles normales créées en 1836 avaient permis, jusqu'à leur fermeture définitive en 1842, la diplomation de seulement quatre normaliens mais de dix-sept normaliennes. Certaines normaliennes enseignaient encore durant les années 1840 et 1850 et, au dire de Meilleur, elles «tenaient admirablement bien les écoles qui leur étaient confiées ${ }^{72}$ ».

Il est difficile d'évaluer avec précision la compétence des instituteurs et des institutrices durant la décennie 1840. La loi scolaire de 1841, qui rétablit un système d'enseignement public, ne précisa que très peu les qualifications requises chez les maîtres, si ce n'est qu'ils devaient avoir «bon caractère» et avoir été «examinés» par des commissaires sur leurs connaissances et leurs capacités. Mais les commissaires étaient souvent illettrés, comme le constate l'inspecteur Cléophe Cimon après sa tournée dans les écoles du comté de Saguenay: «N'est-il pas malheureux de voir que dans le siècle où nous vivons, nos écoles soient conduites par des commis, qui pour la plupart ne savent ni lire ni écrire. Il me fait peine de mentionner que dans certaines municipalités, l'on n'a pu avoir un président, parmi les commissaires qui pût signer son nom ${ }^{73}$.»

Craignant également de s'aliéner les parents et les contribuables durant la «guerre des éteignoirs», les commissaires, comme d'ailleurs les visiteurs ecclésiastiques ou laïques, ne pénétrèrent que bien peu dans les écoles pour s'enquérir des progrès accomplis par les écoliers ou assister aux examens publics ${ }^{74}$. Quant au surintendant Meilleur, il ne pouvait à lui seul se charger de l'inspection des écoles. Après l'adoption de la loi de 1846 qui créait les premiers bureaux d'examinateurs, l'un à Québec, l'autre à Montréal, Meilleur spécifia clairement les qualifications que les commissaires devaient exiger des instituteurs qu'ils comptaient engager pour les écoles élémentaires. Désormais, en plus de savoir montrer à lire,

72. Louis-Philippe Audet, Le système scolaire de la province de Québec (Québec, Les Éditions de l'Érable, 1956), 6: 157-174.

73. Rapport de novembre 1852 (JALPC 1853, App. J.J.). L'inspecteur John Bruce est également très critique à leur égard, notamment dans son rapport du 28 octobre 1853 (JALPC 1854-1855, App. B.). Voir également le rapport de l'inspecteur Césaire Germain du $1^{\text {er }}$ janvier 1853 (JALPC 1856, App. 16.). App. J.J.).

74. Voir à ce sujet le rapport de l'inspecteur Rotus Parmelee du 21 juillet 1852 (JALPC 1853, 
à écrire et à compter, les instituteurs et les institutrices devaient pouvoir enseigner correctement «l'arithmétique jusqu'à la règle de trois inclusivement», de même que les éléments de la grammaire et de la géographie «à commencer par celle du Canada ${ }^{75} \gg$. Mais la vérification de ces connaissances était de nouveau laissée aux commissaires.

En 1849, lorsque la loi exigea des instituteurs qu'ils obtiennent leur brevet avant juillet 1852, Meilleur insista également sur l'obligation qu'ils avaient en outre de posséder une bonne méthode d'enseignement, par laquelle ils inculqueraient aux enfants «d'une manière systématique et graduée, suivant leur âge et leur degré d'avancement, les principes des connaissances usuelles ${ }^{76}$ ». C'était beaucoup demander à des enseignants qui ne disposaient ni de formation dans une école normale ni de manuels ou journaux pédagogiques. Aussi les inspecteurs devaient-ils constater la compétence incertaine des instituteurs, comme le révèle ce commentaire de l'inspecteur Magloire Lanctôt, rédigé après sa première tournée des écoles de Huntingdon et de Beauharnois: «Il se rencontre des instituteurs habiles; mais ils sont rares, si toutefois il est permis d'en juger par l'examen des élèves; car la presque totalité possède des diplômes, et je n'ai pas le droit de les examiner ${ }^{77}$.»

Après sa visite de novembre 1852, l'inspecteur Cléophe Cimon écrivit quant à lui: «Je connais plusieurs instituteurs qui ont obtenu des diplômes qui ne les méritent pas [sic]; ils ont certainement trompé le bureau $^{78}$.» La détention d'un brevet n'était pas la garantie d'une compétence assurée. Dans un mémoire adressé au Comité d'enquête Sicotte, l'avocat Jacques Crémazie, membre du Bureau d'examinateurs de Québec, devait d'ailleurs confirmer l'indulgence de ce bureau, lequel avait accordé des brevets à un grand nombre de candidats non qualifiés, de crainte de voir fermer autant d'écoles ${ }^{79}$. Un jugement sévère que Meilleur tenta d'atténuer: les commissaires avaient dans certains cas engagé des maîtres «non convenablement qualifiés» mais toujours, selon lui, «ces instituteurs et ces institutrices étaient assez instruits pour répondre au besoin actuel des enfants, eu égard à leur âge et à leur degré d'avancement ${ }^{80}{ }$.

\footnotetext{
75. Circulaire $\mathrm{n}^{\circ}$ 9, datée du 15 juin 1846 (JALPC 1846, App. P).

76. Circulaire $\mathrm{n}^{\mathrm{O}} 12$, du 4 juin 1849 (JALPC 1850, App. U).

77. Rapport du 29 juillet 1852 (JALPC 1853, App. J.J.).

78. JALPC 1853, App. J.J.

79. Ce mémoire est repris en annexe du rapport Sicotte (JALPC 1853, App. J.J.).

80. Rapport du 20 avril 1853 (JALPC 1853, App. J.J.).
} 
Qu'en est-il des institutrices exemptées, elles, du brevet? On a pu constater plus haut les talents de certaines parmi les plus jeunes. Durant la décennie 1850 , les inspecteurs mentionnèrent fréquemment la présence d'institutrices formées dans des couvents, des académies ou des écoles modèles, dont ils disaient le plus grand bien ${ }^{81}$. D'ailleurs, André LabarrèrePaulé devait lui-même écrire à propos du personnel enseignant des années 1850: «La bonne marche des écoles n'est pas nécessairement en relation avec l'âge, le sexe ou la possession d'un brevet.» Dans certains comtés, ajoutait-il, les institutrices étaient plus compétentes et plus appréciées que les instituteurs ${ }^{82}$. Bien sûr, il se trouvait des institutrices dont les compétences laissaient à désirer ${ }^{83}$. C'est ce que constata, par exemple, en juillet 1852 , l'inspecteur John Bruce à propos de l'institutrice de l'école de l'arrondissement $\mathrm{n}^{\mathrm{o}} 11$ de la paroisse de Saint-Jean-Chrisostome: «Elle [l'école] est dirigée par une femme de connaissances bien ordinaires, et qui paraît manquer de la vivacité et du caractère actif que doit avoir une institutrice... elle n'enseigne qu'un petit nombre de branches ${ }^{84}$.» En ce qui concerne l'institutrice de l'école $\mathrm{n}^{\mathrm{O}} 9$ du township de Chatam, l'inspecteur Bruce écrit: «son enseignement est de peu de valeur. Les branches qu'elle enseigne sont en petit nombre et peu bien $[\mathrm{sic}]$ exposées $^{85}$.»

En somme, la compétence et l'incompétence en matière d'enseignement existaient assurément tant chez les unes que chez les autres. Mais on ne peut accuser les enseignantes de discréditer davantage leur métier que les instituteurs.

\section{Et intéressées à leur métier}

L'accusation répétée de passivité et d'inertie que porte André Labarrère-Paulé à l'endroit des institutrices rurales mérite également d'être réévaluée. Selon mes premières recherches sur les maîtresses d'école indépendantes de Montréal du premier tiers du XIX ${ }^{\mathrm{e}}$ siècle, les dirigeantes de ces écoles, institutrices réputées faisant carrière dans l'enseignement depuis maintes années et sans doute fort appréciées de la population et conscientes de leurs capacités, exigeaient des frais de scolarité souvent élevés et en moyenne légèrement supérieurs à ceux demandés par leurs collè-

81. Voir, entre autres, les rapports, pour l'année 1856, des inspecteurs Georges Tanguay et Georges-Allan Bourgeois (JALPC 1857, App. 58) et celui, pour l'année 1858, de l'inspecteur Jean Crépault (JALPC 1859, App. 58).

82. André Labarrère-Paulé, Les instituteurs..., 163, 166.

83. Voir les rapports des inspecteurs John Bruce et Jean Crépault pour l'année 1852 (JALPC 1853, App. J.J.).

84. Rapport du 27 août 1852 (JALPC 1853, App. J.J.).

85. Ibid. 
gues masculins ${ }^{86}$. Les recensements scolaires de Viger ne permettent pas d'apprécier davantage la perception qu'ont de leur métier et de leur propre valeur les institutrices rurales durant la période 1825-1850. Encore une fois, les témoignages des inspecteurs d'école nous apportent des indices.

Ceux-ci mentionnèrent à plusieurs reprises que les institutrices rurales réussissaient très bien parce qu'elles faisaient preuve, même les toutes jeunes, d'un vif désir d'améliorer leurs connaissances et leur méthode d'enseignement. Citons l'inspecteur Bruce à propos de l'institutrice de l'école de l'arrondissement $\mathrm{n}^{\mathrm{O}} 3$ de Lachute: «Considérant les connaissances limitées de cette institutrice et le peu d'occasions qu'elle a de se mettre au fait des progrès dans l'art de l'enseignement, sa persévérance et ses efforts lui font certainement beaucoup d'honneur ${ }^{87}$.»

Les institutrices n'avaient en effet que bien peu d'occasions de se perfectionner. Elles étaient exclues des premières associations d'instituteurs mises sur pied par Meilleur et Chauveau et des conférences organisées par les écoles normales Jacques-Cartier (Montréal) et Laval (Québec) ${ }^{88}$. D'ailleurs, lorsque furent établies ces écoles en 1857, les femmes n'étaient admises qu'à la seule section féminine de l'École normale Laval, confiée aux ursulines de Québec ${ }^{89}$. Sans l'affirmer bien sûr, on pourrait par ailleurs soupçonner le surintendant Meilleur de ne pas s'être empressé de communiquer aux institutrices Le Guide de l'instituteur de François-Xavier Valade, préparé à l'intention des candidats aux examens des bureaux d'examinateurs, examens dont il avait exempté les femmes ${ }^{90}$. Malgré cette exemption, 23 femmes s'étaient présentées volontairement à de tels examens, selon le comité Sicotte. Un fait d'autant plus notable que le Bureau d'examinateurs de Montréal refusait de «procéder à l'examen des personnes

86. Andrée Dufour, «Les maîtresses d'écoles indépendantes: une contribution essentielle à l'éducation de la jeunesse montréalaise du premier tiers du XIX ${ }^{\mathrm{e}}$ siècle», dans Evelyne Tardy et al., Les Bâtisseuses de la Cité (Montréal, ACFAS, 1993), 125-139; voir également Andrée Dufour, «Mme A. Trudeau: une institutrice à la tête d'une école indépendante», dans Maryse Darsigny et al., Ces femmes qui ont bâti Montréal (Montréal, les Éditions du remue-ménage, 1994), 71-72, ainsi que la communication Le corps enseignant..., déjà citée.

87. Rapport pour l'année 1855 (JALPC 1856, App. 16). Voir aussi les rapports pour l'année 1852 des inspecteurs Bruce, Germain et Lanctôt (JALPC 1853, App. J.J.).

88. André Labarrère-Paulé, Les instituteurs..., 223-224.

89. La section féminine de l'École normale Jacques-Cartier ne sera ouverte qu'en 1899 chez les dames de la Congrégation. Marta Danylewycz a souligné le fait que les institutrices étaient laissées à elles-mêmes dans «Sexes et classes sociales dans l'enseignement: le cas de Montréal à la fin du $19^{\mathrm{e}}$ siècle», dans Nadia Fahmy-Eid et Micheline Dumont, dir., Maîtresses de maison, maîtresses d'école. Femmes, famille et éducation dans l'histoire du Québec (Montréal, Boréal Express, 1984): 108-109.

90. Dans Les instituteurs laïques..., 139, André Labarrère-Paulé écrit d'ailleurs: «L'élément féminin est donc dans l'enseignement considéré comme secondaire: [...] Meilleur n'accordera vraiment d'importance qu'au personnel masculin.» 
du sexe ${ }^{91} \gg$. Lorsque, en 1856, la loi obligea les institutrices à décrocher leur brevet dans un délai d'un an, elles se présentèrent en grand nombre aux nouveaux bureaux de comté accessibles aux hommes et aux femmes. Aussi, dès 1857 , elles présentèrent le même pourcentage de diplomation que les instituteurs: $59 \%$. Deux ans plus tard, les rapports du surintendant révèlent que 626 des 976 instituteurs (65\%) et 1338 des 2129 institutrices (63\%) possédaient un brevet d'enseignement. Mais, de quel type de brevet s'agitil? Et où ces brevets ont-ils été acquis? Les tableaux 5 et 6 de même que le tableau 1 , fournissent des réponses à ces questions.

TABLEAU 5

RÉPARTITION DES BREVETS ACCORDÉS PAR LES BUREAUX D'EXAMINATEURS DE 1847 À 1859, SELON LE GENRE

\begin{tabular}{lcccc} 
Type de brevet & \multicolumn{2}{c}{ Hommes } & \multicolumn{2}{c}{ Femmes } \\
& Nombre & $\%$ & Nombre & $\%$ \\
Élémentaire & 781 & 82,5 & 2133 & 97,3 \\
Modèle & 143 & 15,1 & 58 & 2,7 \\
Académique & 23 & 2,4 & 1 & 0,1 \\
Total & 947 & - & 2192 & -
\end{tabular}

Source: Rapport du surintendant de l'instruction publique du Bas-Canada pour 1859 (JALPC 1860, App. 50).

TABLEAU 6

RÉPARTITION DES DIPLÔMES ACCORDÉS PAR LES ÉCOLES NORMALES EN 1858 ET 1859, SELON LE GENRE

Type de diplôme

\begin{tabular}{cc}
\multicolumn{2}{c}{ Hommes } \\
Nombre & $\%$ \\
35 & 43,2 \\
46 & 56,8 \\
0 & 0 \\
81 &
\end{tabular}

\begin{tabular}{rc}
\multicolumn{2}{c}{ Femmes } \\
Nombre & $\%$ \\
82 & 61,1 \\
46 & 35,9 \\
0 & 0 \\
128 &
\end{tabular}

Source: Rapport du surintendant de l'instruction publique du Bas-Canada pour 1859 (JALPC 1860, App. 50).

91. Rapport de l'inspecteur Antoine-P. Consigny du 10 août 1852 (JALPC 1853, App. J.J.). 
Il se dégage en premier lieu que $92,1 \%$ et $95,5 \%$ des permis d'enseignement décernés aux hommes et aux femmes l'avaient été par l'intermédiaire d'un bureau d'examinateurs ${ }^{92}$. Ce constat n'étonne guère, compte tenu du grand nombre de bureaux disponibles et de l'ouverture toute récente des écoles normales. Les tableaux 5 et 6 montrent surtout une tendance plus forte chez les hommes à solliciter des brevets modèles et académiques, alors que les femmes, tout particulièrement celles qui passaient par les bureaux, s'en tenaient davantage au simple brevet d'école élémentaire. Cette tendance s'amplifiera d'ailleurs nettement durant les décennies suivantes ${ }^{93}$. La carrière que pouvaient espérer la majorité des institutrices rurales était bien courte et les perspectives d'avancement qui leur étaient offertes dans l'appareil scolaire étaient généralement bien faibles ${ }^{94}$. À ces facteurs explicatifs, on peut ajouter le maintien des bureaux d'examinateurs régionaux et, surtout, l'existence d'une seule école normale ouverte aux aspirantes enseignantes francophones. On observe en effet que l'écart entre les aspirations des hommes et des femmes s'amenuise fortement lorsque l'école normale est le lieu d'obtention du permis. Ainsi, 32 hommes et 48 femmes s'étaient inscrits à l'École normale Laval en 1858-1859. Quatorze hommes, 44\% des normaliens, étaient candidats au diplôme d'écoles modèles; vingt-trois femmes, $48 \%$ des normaliennes, aspiraient à un tel brevet ${ }^{95}$. Lorsqu'une deuxième puis une multitude d'écoles normales de filles ouvriront leurs portes à partir de 1899 et que, la même année, les bureaux d'examinateurs de comté fermeront au profit du seul Bureau central des examinateurs, les femmes se contenteront de moins en moins du brevet élémentaire ${ }^{96}$.

Soulignons un dernier élément révélateur de l'intérêt des institutrices vis-à-vis de leur métier: l'abonnement à la revue pédagogique créée en 1857 par Chauveau, le Journal de l'instruction publique. Entre les mois de septembre 1858 et de décembre 1859 , nous avons trouvé, parmi la correspondance dépouillée, 36 demandes d'abonnement à la revue. Dix provenaient d'instituteurs, dix d'ecclésiastiques, une d'un établissement et quinze d'institutrices. Certes, ces dernières sont plus nombreuses que les premiers à enseigner, mais il nous apparaît néanmoins difficile encore ici de les taxer d'indifférence et d'inertie à l'égard de leur métier.

92. Ce résultat est obtenu en additionnant le nombre total de brevets décernés par les bureaux d'examinateurs et par les écoles normales de 1847 à 1859.

93. Voir la répartition du personnel enseignant selon le sexe et le niveau de qualification qu'ont établie M'hammed Mellouki et François Melançon dans Le corps enseignant du Québec de 1845 à 1992. Formation et développement (Montréal, Les Éditions Logiques, 1995), 304.

94. Marta Danylewycz, «Sexes et classes sociales...», 94.

95. Rapport de Jean Langevin, directeur de l'École normale Laval, du 15 août 1859 (JALPC 1960, App. 50).

96. C'est ce que révèle la répartition du personnel enseignant signalée à la note 93. 


\section{CONCLUSION}

Comme l'estimaient déjà une majorité d'historiennes et d'historiens, la féminisation du corps enseignant au Québec s'est produite durant la première moitié du XIX ${ }^{\mathrm{e}}$ siècle. Cette étude fait même apparaître une majorité de femmes dans l'enseignement primaire dès avant 1840 . On ne peut nier, dans ce processus particulièrement hâtif en Amérique du Nord, le rôle joué par la baisse de la rémunération des enseignants et, surtout, des enseignantes. Une baisse qui tire son origine bien au-delà de «l'avarice» des commissaires et de «linertie» des jeunes institutrices. Le désengagement de l'État du paiement des maîtres, la perception concernant le travail féminin rétribué et les difficultés vécues par maintes collectivités en voie d'établissement expliquent également cette diminution. Par ailleurs, le fréquent manque d'intérêt des hommes instruits pour l'enseignement primaire ainsi que l'affirmation croissante de l'Église furent autant de facteurs à l'origine de la féminisation du métier. Mais les maigres salaires consentis aux institutrices rurales et le peu d'empressement des autorités scolaires à reconnaître leur contribution à la diffusion des connaissances ne firent pas de celles-ci des créatures inertes et indifférentes pour autant. Manifestant souvent beaucoup d'intérêt pour leur métier, un métier dans lequel elles trouvaient également une occasion de gagner leur vie, de faire carrière ou encore de tirer avantage, en vue d'un mariage futur, d'une formation souvent jugée pertinente, les institutrices rurales se consacrèrent en grand nombre et en tous lieux à l'éducation des jeunes enfants.

Cette étude ne prétend aucunement avoir tracé une image complète et définitive des enseignantes de campagne bas-canadiennes. Leur attitude, comme d'ailleurs celle des instituteurs, doit être davantage documentée. En effet, les sources dépouillées ici ne permettent de la saisir, la plupart du temps, qu'à travers les témoignages des commissaires et, surtout, des inspecteurs d'école. Il faudrait lire les témoignages des institutrices elles-mêmes pour mieux connaître leurs sentiments et leurs comportements à l'égard d'un métier qu'elles s'approprient au XIX ${ }^{\mathrm{e}}$ siècle. Un dépouillement élargi de la correspondance qu'elles adressèrent aux élus locaux et aux autorités scolaires devrait permettre cette analyse. En attendant, force est de constater qu'elles participèrent largement et d'une façon généralement convenable à l'entreprise de scolarisation de l'ensemble de la jeunesse bas-canadienne. Enfin, il m'apparaît tout aussi essentiel de revenir sur le désengagement constaté de l'État concernant la rétribution des maîtres. Un désengagement dont la portée a peut-être été sous-estimée et qui se révèle de première importance dans le processus de féminisation du corps enseignant bas-canadien. 